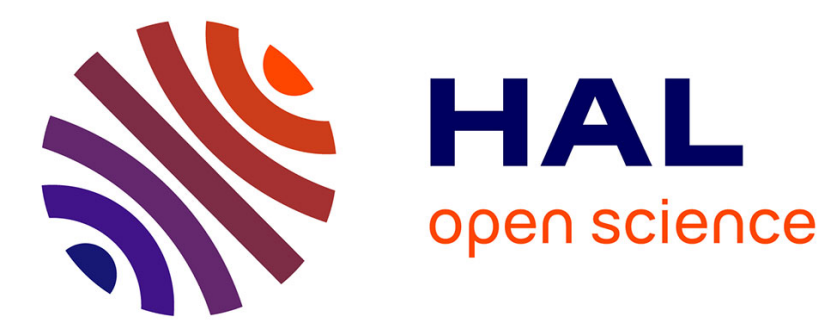

\title{
Finance et accumulations dans le capitalisme post-industriel
}

Bernard Paulré

\section{- To cite this version:}

| Bernard Paulré. Finance et accumulations dans le capitalisme post-industriel. 2008. hal-00223912

\section{HAL Id: hal-00223912 \\ https://hal.science/hal-00223912}

Preprint submitted on 29 Jan 2008

HAL is a multi-disciplinary open access archive for the deposit and dissemination of scientific research documents, whether they are published or not. The documents may come from teaching and research institutions in France or abroad, or from public or private research centers.
L'archive ouverte pluridisciplinaire HAL, est destinée au dépôt et à la diffusion de documents scientifiques de niveau recherche, publiés ou non, émanant des établissements d'enseignement et de recherche français ou étrangers, des laboratoires publics ou privés. 


\title{
FINANCE ET/ACCUMULATIONS DANS LE CAPITALISME POST-INDUSTRIEL
}

\author{
Bernard Paulré \\ Isys-Matisse, CES, UMR Paris 1 - CNRS n 8595 \\ Ecole d'économie de Paris
}

Depuis les années 1970, et avec la crise du fordisme, le capitalisme est entré dans une phase de mutation profonde. Nous pensons que la sortie du capitalisme industriel est la caractéristique majeure de cette période et qu'est en train d'émerger ce que nous appelons un capitalisme cognitif. Selon d'autres analystes, c'est en fonction du rapport à la finance que cette nouvelle période du capitalisme devrait être caractérisée. L'importance manifeste prise par l'accumulation financière explique en partie l'origine du problème et son enjeu. Opposant l'accumulation "réelle" (i. e. l'investissement en capital fixe) et l'accumulation financière (i. e. le placement en titres financiers), ces analystes considèrent que les taux de rendement sont structurellement favorables à la seconde. Dans ces conditions nous devrions caractériser le capitalisme contemporain de financier, c'est-à-dire à partir du type d'accumulation apparemment dominant.

A l'examen, ce n'est pas aussi simple, et nous rejetons l'alternative qui nous mettrait en demeure de choisir, pour qualifier le capitalisme contemporain, entre un capitalisme cognitif et un capitalisme financier. Le problème auquel nous sommes confrontés n'est pas de choisir entre les deux thèses, mais plutôt de mieux comprendre les interdépendances entre les mutations de la connaissance et de la finance dans l'époque post-industrielle. C'est naturellement à partir de l'étude des conditions de l'accumulation que nous allons poser les problèmes.

\section{1- Les nouvelles formes d'accumulation du capitalisme cognitif}

Par capitalisme cognitif nous désignons une configuration caractérisée par un passage au post-industrialisme, dans laquelle les capitalistes s'efforcent, et ils y parviennent, à s'approprier une partie de la valeur produite par le travail, alors que celui-ci, désormais centré sur la connaissance, en un sens large, se manifeste sous des formes nouvelles et diffuses. Cette configuration est donc caractérisée par une tension centrale et une ambiguïté. Alors que les formes traditionnelles de la production capitaliste s'érodent, sinon disparaissent ou se transforment sensiblement, les capitalistes cherchent à maintenir leur contrôle et à capter la valeur, parfois en préservant des dispositifs de valorisation conçus initialement pour la période fordiste. Et alors que les modes de vie et de travail ont profondément changé, ont encore cours des représentations, des comportements et des interactions façonnés naguère par le fordisme.

Les mutations caractéristiques du capitalisme cognitif ont été identifiées et discutées depuis 2000, année de la première présentation de la thèse. Il existe aujourd'hui des interprétations assez variées en dépit, le plus souvent, d'une base et d'une origine communes. Des thématiques majeures ont été identifiées et mises en avant par des auteurs qui manifestent parfois fortement leur conviction quant à la caractéristique essentielle de cette configuration. Mais ce n'est pas pour autant qu'elles doivent être considérées comme des options alternatives. La recherche sur le capitalisme cognitif s'inscrit dans le contexte d'un processus historique en cours, et la diversité des approches est le reflet de la complexité des expériences et des mutations observées. La démarche en est d'ailleurs heuristique et collective. 
L'orientation sans doute la plus fondamentale et englobante concerne la nouvelle nature du travail productif et les transformations de l'organisation du travail. La première distinction que l'on doit faire dans ce domaine porte sur : d'une part la tendance du travail à devenir de plus en plus immatériel ; et d'autre part la dimension sociale et "immédiatement communicante" du travail vivant. Les aspects immatériels du travail, de la production et de la consommation (individuelle comme collective) ont retenu dès l'origine l'attention au point que, selon certains auteurs, le capitalisme cognitif serait caractérisable sur cette base, en y associant parfois le développement des TIC ${ }^{1}$. Pour d'autres, c'est la place de l'incertitude, de la flexibilité et le rôle des interactions langagières dans ce contexte qui sont considérés comme caractéristiques de la nouvelle organisation du travail. Ce sont en effet les interventions mobilisant des savoirs, des compétences (c'est-à-dire de l'expérience) et la réactivité qui deviennent déterminantes face aux événements et aux imprévus d'autant plus stratégiques que la programmation et la planification occupent une place importante. On assisterait à un "tournant linguistique" 2 .

Le point le plus significatif est sans doute que l'on doit désormais fondre le travail dans tout le champ des relations sociales. Il est toujours au centre de la production mais tout est production, et le travail cognitif est lié étroitement au temps de la vie. On ne peut donc en rester à la conception traditionnelle du travail, limitée aux périodes de temps salarié ainsi qu'aux lieux dédiés à la production. Il ne faut d'ailleurs pas considérer exclusivement le travail intellectuel et "cognitif". Il faut tenir compte aussi du corps et des affects.

La compréhension historique des mutations du travail et de son organisation peut ainsi s'appuyer sur la thèse du passage du régime disciplinaire au régime de contrôle ${ }^{3}$. La société de contrôle se caractérise par "une intensification et une généralisation des appareils normalisant de la disciplinarité... mais, au contraire de la discipline, ce contrôle s'étend au delà des sites structurés des institutions sociales, par le biais de réseaux souples, modulables et fluctuants" ${ }^{4}$. Dans cette société, un nouveau paradigme de pouvoir se manifeste. La vie faisant désormais partie du champ du pouvoir, celui-ci devient entièrement biopolitique. Il représente à la fois "l'extension de la contradiction économique et politique sur tout le tissu social [et] l'émergence de la singularisation des résistances dont il est en permanence traversé" 5

Les deux types d'approches évoquées se rejoignent, tout en restant distinguables, sur le terrain de ce que A. Négri appelle "l'excédence". D'un côté, il y a excédence parce que la valeur est le produit d'une action créatrice. De l'autre, l'excédence résulte de la "démesure" : si le travail cognitif est lié au temps de la vie, "ses produits sont des produits de liberté et d'imagination" 6 . Nous sommes dans un procès de "destruction créatrice", en donnant évidemment à cette notion une portée qu'elle n'avait certainement pas chez J. Schumpeter.

La question de l'excédence nous conduit au thème de l'accumulation dans le capitalisme cognitif. Ce sont les produits de la créativité, intellectuels ou affectifs, qui deviennent prééminents, l'accumulation de biens d'équipement et d'organisation devenant secondaire. De plus, l'accumulation se réalise souvent directement là où se réalise la production et se présente sous des formes originales. D'abord à cause de son objet : immatériel le plus souvent. Ensuite parce qu'il y a souvent, mais pas toujours, dissociation de l'accumulation effective, réelle, cognitive et affective, et de l'accumulation monétaire. L'accumulation n'implique plus nécessairement un investissement monétaire. Elle peut être réalisée par les salariés eux mêmes. Enfin, alors que l'accumulation reposait ou s'articulait auparavant avec les investissements

\footnotetext{
1 Technologies de l'information et de la communication.

2 C. Marazzi, Et vogue l'argent, Ed. de l'Aube, 2003.

3 G. Deleuze, Pourparlers, Ed. de Minuit, 1990.

M. Hardt et A. Negri, Empire, Exils Editeurs, 2000.

5 A. Négri, La fabrique de porcelaine, Stock, 2006.

${ }^{6}$ Ibid.
} 
matériels dans l'entreprise, maintenant l'accumulation est diffuse. La capacité créatrice se réalise partout, y compris en dehors des entreprises. La productivité est directement sociale. Mais il n'y pas seulement amélioration de l'efficience. Il y a aussi créativité et valeurs d'usages nouvelles c'est-à-dire innovations en tous genres. Provenant aussi bien des producteurs que des consommateurs. Cette dimension innovatrice est importante, certains analystes allant jusqu'à considérer que le capitalisme cognitif se manifeste par un processus d'innovation permanente.

L'accumulation dont il est généralement question dans le contexte du capitalisme cognitif est une accumulation "réelle", en ce sens qu'elle ne porte pas sur des titres ou des créances. Elle est principalement immatérielle. Lorsqu'elle est associée à une accumulation matérielle, celle-ci ne se réalise pas nécessairement à proximité. On peut rapprocher cela du fait que, dans le cadre des restructurations industrielles caractérisant le postfordisme, l'une des priorités est celle de l'entreprise "mince" ou "légère" c'est-à-dire, entre autres, de l'entreprise dont les immobilisations augmentent le moins possible, voire régressent. Les externalisations contribuent à réaliser cet allégement en capital fixe. L'accumulation "cognitive" est conforme avec cette orientation, quoique les raisons en soient en partie différentes.

\section{2- La financiarisation contemporaine}

L'idée d'une financiarisation des économies est justifiée par un certain nombre de transformations comme, par exemple :

- l'accroissement important des flux financiers au plan international et domestique,

- l'importance accrue du rôle et, plus généralement, de l'influence des acteurs financiers,

- l'endettement accru des ménages et une dynamique de la consommation qui repose de plus en plus sur le crédit,

- l'endettement accru des entreprises qui ne recourent plus aux émissions d'actions nouvelles puisque, au contraire, on assiste à des rachats systématiques d'actions,

- la place de la détention d'actifs financée par l'endettement,

- l'accroissement des revenus "rentiers",

- la jeu de l'effet de richesse ${ }^{7}$,

- l'affirmation de la primauté de la "valeur pour l'actionnaire" et un débat sur la gouvernance des entreprises,

- les conséquences des pressions financières sur les stratégies des firmes qui, de façon schématique, sont passées d'une logique fordienne ("garder les profits et investir") à une logique que l'on peut résumer par le nouvel impératif : "diminuer la taille et distribuer" ${ }^{8}$.

L'accumulation financière occuperait désormais une position centrale. La question que nous posons est celle du sens de cette évolution. S'agit-il d'un phénomène autonome qui a son origine dans un événement peut être contingent et qui s'affirme finalement dans une relation de domination ou de priorité vis-à-vis de ce que les économistes appellent la "sphère réelle" ? Ou bien ce phénomène ne peut-il être compris que dans un processus de co-évolution avec les transformations du secteur réel, et donc comme élément de l'émergence systémique du postfordisme ? Nous allons montrer ici les limites d'une approche de la finance comme pôle d'accumulation autonome.

Commençons par ce qui apparaît comme la décision financière majeure, c'est-à-dire le virage de 1979 aux Etats-Unis ${ }^{9}$. Cette décision est inaugurale et cruciale. On va passer d'un système de taux d'intérêt faibles à un système à taux d'intérêt réels positifs. Par ailleurs, tout

\footnotetext{
7 C'est-à-dire l'effet de la richesse détenue sur la consommation.

${ }^{8}$ W. Lazonick and M. O'Sullivan (eds.), Corporate Governance and Sustainable Prosperity, Palgrave, 2002.

9 Moins de deux mois après sa nomination à la tête de la FED, P. Volcker augmenta fortement le taux des fonds fédéraux, au point que les taux réels devinrent positifs à la hauteur de presque 3,5\%
} 
en basculant dans le postfordisme, des comportements et des mentalités héritées du fordisme subsistent. Plus particulièrement, alors que le contexte est déflationniste, les anticipations inflationnistes se maintiennent. Ce qui contribue à entretenir un sentiment d'incertitude. Accompagné ou aggravé par l'incertitude liée à la globalisation et celle, de nature plus industrielle quoique pas uniquement, liée aux changements technologiques. C'est dans ce contexte que les marchés financiers commencent, dans les années 80, à se développer considérablement, constituant apparemment une menace et une concurrence pour l'accumulation réelle.

Lorsque C. Marazzi écrit que la "sortie du fordisme part du haut du système monétaire US" $^{10}$, il faut l'interpréter strictement : l'acte "officiel" qui engage ou signale la sortie du fordisme part du haut, mais ce n'est ni la crise du fordisme ni, à supposer qu'elle soit séparable, la crise financière qui l'accompagne qui partent du haut. Si la décision de P. Volcker semble suivre la crise du fordisme, les facteurs de la crise financière en font partie intégrante ${ }^{11}$. La déstabilisation financière n'est pas postérieure à la crise du fordisme, elle en fait partie. Si le virage de 1979 est inaugural par rapport à ce qui suit, il est fortement contraint et virtuellement engagé par la crise, inscrite elle-même dans la nature du fordisme. La finance n'est pas dominante au sens ou elle aurait une sorte d'exogénéité ou de primauté absolue. S'il y a une primauté, c'est éventuellement celle du politique qui l'instrumentalise.

Il est frappant d'observer, presque trente ans plus tard, qu'il n'existe pas de consensus sur ce que désigne précisément la notion de financiarisation. Surtout si on l'aborde par le côté empirique : les manifestations en sont suffisamment variées pour justifier bon nombre de points de vue différents. C'est pourquoi certains auteurs proposent une définition que l'on qualifiera de large. Comme G. Epstein, qui désigne par financiarisation "le place croissante des motifs financiers, des marchés financiers, des acteurs et des institutions financiers dans le fonctionnement des économies nationales et internationale" ${ }^{12}$.

Nous suggérons un autre type de définition, plus qualitatif : il y a financiarisation dès lors que les logiques financières prennent le pas sur les logiques économiques, c'est-à-dire lorsque les comportements sont moins focalisés sur des valeurs de flux économique que sur l'évolution des postes du bilan patrimonial. La financiarisation se traduit par une "patrimonialisation" des comportements. Ce qui s'accorde bien avec ce que l'on observe, c'està-dire avec la financiarisation de la relation salariale et celle des entreprises. La financiarisation est un phénomène diffus qui se traduit, au plan macroéconomique, par l'émergence d'une asset economy.

Selon une interprétation qui s'accorde bien avec la définition proposée nous serions entrés dans un "régime patrimonial", caractérisé par "le rôle désormais prépondérant que jouent les marchés des actifs... dans la détermination des équilibres macroéconomiques [et par] l'extension de l'actionnariat salarié via l'importance des investisseurs institutionnels dans la finance et la gouvernance des entreprises comme instance primordiale de la régulation" ${ }^{13}$. L'accent est plus particulièrement mis sur le rôle central des marchés d'actifs et le poids des investisseurs institutionnels. Le type de modèle proposé pour représenter cette configuration fait cependant apparaître une forte fragilité. Ce qui suggère que la prépondérance du marché financier n'est pas un phénomène spontanément durable. On montre d'ailleurs que, dans un régime tiré par la finance, celle-ci doit être considérée comme un élément du système complexe qui l'englobe ${ }^{14}$.

\footnotetext{
${ }^{10}$ C. Marazzi, op. cit.

11 Souvenons nous de la locution latine et de l'erreur logique qu'elle stigmatise : Post hoc, ergo propter hoc.

12 G. Epstein, Financialization and the World Economy, Edward Elgar, 2005.

13 M. Aglietta, Le capitalisme de demain, Fondation Saint-Simon, 1998.

14 R. Boyer, Is a finance-led growth regime a viable alternative to Fordism ?, Economy and Society, $2000, \mathrm{n}^{\circ} 1$.
} 
Or c'est bien l'avènement d'une financiarisation néo-libérale marchande qui constitue l'un des traits caractéristiques du postfordisme. Les transformations opérées concernent autant le développement et la complexification de la finance que le changement du lieu où elle opère principalement, et du pôle d'où elle exerce son emprise. Ce qui est en jeu c'est l'exercice d'une accumulation qui se réalise dans un espace marchand, encadré institutionnellement par un ensemble de règles, qui fait intervenir des acteurs professionnels et qui aurait vocation a fonctionner, dit-on, de façon transparente. M. Aglietta avance une explication qui repose sur l'affirmation de la primauté et de la dominance foncière de la finance : elle "oriente en très longue période la développement du capitalisme. Elle détermine les conditions de financement qui, alternativement, entraînent des phases longues où la croissance est encouragée, puis découragée" ${ }^{15}$. L'évolution du capitalisme serait rythmée par deux modes de régulation financière : les systèmes financiers à structure administrée d'une part, la finance libéralisée qui "favorise une inflation stable et basse, mais entrave l'accumulation" d'autre part. Malgré son intérêt, cette analyse a le défaut commun à toutes les interprétations cycliques : celui du fatalisme et du caractère automatique des transformations. Elle ignore les forces motrices d'un changement considéré comme une vraie rupture et non comme un simple retour. Il est difficile de poser a priori la finance en surplomb de la sphère réelle.

Selon un autre point de vue assez répandu, la finance exercerait une emprise sur le système industriel, tout en étant déconnectée des phénomènes "réels". Elle effectuerait une ponction préalable sur le profit et contraindrait fortement l'investissement. Bref, le capital financier serait parasite. Nous ne pensons pas que cette interprétation soit pertinente. On peut observer, d'abord, que le profit englobe le profit industriel et le profit financier. Si bien qu'en deçà de la rivalité entre capitalistes, la logique capitaliste d'exploitation reste la même ${ }^{16}$. Cette approche est critiquable également car on peut montrer que le placement de l'épargne en finance n'est pas le résultat d'une contrainte préalable pesant sur l'affectation des profits, mais la conséquence de l'absence d'opportunités d'investissements rentables ou considérés comme pertinents stratégiquement ${ }^{17}$. Ce que suggèrent le niveau de l'autofinancement et la faiblesse de l'apport d'argent collecté par les entreprises sur les marchés financiers.

Pour toutes ces raisons, nous devons nous engager dans la voie d'une approche conjointe de l'accumulation réelle et de l'accumulation financière en les replaçant toutes deux dans le contexte de la dynamique d'émergence du capitalisme cognitif.

\section{3- La financiarisation et le capitalisme cognitif sont solidaires}

Dans le cadre du fordisme, la sphère financière capte une partie de la valeur créée via les intérêts et les dividendes versés. Si l'on observe parfois des bulles, cela ne s'interprète pas comme de la création de valeur au sens classique du terme, et relève de ce qu'on appelle du capital fictif. La dimension évaluation et cognitive de la sphère financière est seconde car la valeur est créée dans l'entreprise et évaluée dans la sphère productive.

Dans le capitalisme cognitif la valeur est issue de plusieurs sources, internes et externes à l'entreprise, selon des canaux de transmission diffus et en partie immatériels. La production est commune. Il y a exploitation dès qu'un acteur veut s'approprier une partie ou la totalité de celle-ci. Ce qui est en général le cas de l'entreprise. Il n'y a plus de valeur imputable économiquement dans l'entreprise. La fonction évaluative de la sphère financière est centrale.

\footnotetext{
15 M. Aglietta, Macroéconomie financière, La Découverte, 1995.

${ }^{16}$ G. Duménil et D. Levy, La finance capitaliste : rapports de production et rapports de classe, Working Paper, 2006.

17 M. Husson, Le grand bluff capitaliste, La Dispute, 2001.
} 
Plusieurs thèses relatives à la financiarisation dans le postfordisme peuvent être formulées. Toutes mettent en relation la financiarisation et les transformations réelles d'où émerge le capitalisme cognitif.

$\underline{\text { a- La cohérence de l'accumulation immatérielle et de la financiarisation }}$

La question de la relation entre l'accumulation réelle et l'accumulation financière se pose dans un contexte historiquement particulier. On a pu constater en effet un taux d'accumulation (réelle) décroissant ou faible, alors que le taux de profit a tendance à croître et que l'on assiste à une croissance de l'accumulation financière. C'est cette configuration particulière et même inédite que M. Husson baptise précisément de "financiarisation", évoquant "une phase spécifique de 'financiarisation' du capitalisme". La question économique qui se pose n'est donc pas simplement celle de la relation entre accumulation "réelle" et accumulation financière, mais aussi celle de savoir pourquoi l'accumulation réelle est faible, dans une configuration où les profits sont élevés et l'accumulation financière forte.

Pour certains analystes, c'est la faiblesse des opportunités et des occasions (contraintes par les taux de rentabilité désirés et par les politiques de recentrage) qui explique d'abord la faiblesse des investissements et, par voie de conséquence ensuite, la distribution importante de dividendes. Un raisonnement "Kaleckien" ${ }^{18}$ permet de démontrer que la financiarisation (l'accumulation de titres financiers) est la conséquence de l'élévation du taux d'exploitation ${ }^{19}$. Ce qui montre bien au passage que la financiarisation n'est pas un phénomène autonome, qu'elle est au contraire un phénomène endogène et qu'elle doit être étudiée solidairement avec les autres aspects (réels) du fonctionnement de l'économie.

On a observé que, pour des pays importants de l'OCDE et depuis le début des années 80, l'accumulation a décliné alors que les taux de profit ont eu tendance à croître. Un niveau élevé des dividendes versés est associé à un investissement déprimé, et une forte propension à consommer de la part de ceux qui perçoivent ces revenus (les "rentiers") stimule le profit. Une autre façon de justifier le même résultat consiste à observer que le capitalisme refuse de reconnaître et de satisfaire un certain nombre de besoins sociaux : si les occasions d'investissement faiblissent, c'est parce que des besoins ne sont pas identifiés et reconnus comme pouvant et devant être satisfaits. Cet argument débouche sur la question des besoins sociaux selon M. Husson. Ce type de réponse permet d'englober la finance dans une vision systémique donc globale du système économique.

Mais nous pouvons modifier le champ de la question et formuler différemment le problème. Si l'on accepte de considérer que les occasions d'investissements sont limitées du fait que certains besoins sociaux ne sont pas reconnus, on peut tout aussi bien admettre qu'elles semblent limitées parce que certaines activités, qui sont des investissements d'un type original (investissements créatifs individuels, de groupes ou collectifs ; investissements affectifs) ne sont pas reconnues, identifiées et traitées comme tel (ou comme des investissements rentabilisables). Autrement dit, si la logique des opérations économiques s'est modifiée de telle sorte que des bénéfices sociaux apparaissent par d'autres voies, que la productivité s'améliore sans avoir à investir sous la forme classique (ou "fordienne"), on comprend que mécaniquement, économiquement, tout se passera comme s'il y avait sousinvestissement. Cet effet sera clairement renforcé par le caractère immatériel des investissements et la volonté de ne pas alourdir le bilan des entreprises.

On peut alors songer à déplacer l'interrogation initiale : plutôt que de voir dans quelle mesure la financiarisation contraint l'accumulation réelle (matérielle), et puisque celle-ci ne

\footnotetext{
18 Le raisonnement s'appuie sur la distinction entre le comportement des salariés et celui des "rentiers".

19 M. Husson, La théorie des ondes longues et le capitalisme contemporain, Working Paper, 2005.
} 
serait plus la forme dominante de l'investissement, ne vaut-il pas mieux essayer de voir si la financiarisation n'est pas un moyen de contraindre l'accumulation de la puissance créatrice du travail cognitif ou, au moins, d'en orienter l'exercice vers des activités potentiellement rentables dont les effets monétaires positifs peuvent être captés par les capitalistes.

\section{$\underline{\text { b- les marchés financiers, nouvelle forme de gouvernementalité }}$}

Revenons sur la notion de biopolitique qui vise à qualifier la façon dont le pouvoir aborde le gouvernement des populations. Selon M. Foucault, la biopolitique se fonde sur des principes qui spécifient les technologies de gouvernementalité du capitalisme. Elle se manifeste au travers des modes de gestion de l'éducation, de la santé, de l'alimentation, de la sexualité etc. La biopolitique porte sur les aspects vitaux qui sont l'objet des politiques de l'Etat Providence. Durant la période fordiste, c'est l'Etat qui a pris en charge ce que B. Théret appelle le "capital de vie" sur la base d'un principe de solidarité, d'où une alliance vertueuse du fordisme et du welfare state. Par contre, la période qui suit est caractérisée par une crise de l'Etat Providence, par la mise en place de politiques d'individualisation des rémunérations et la privatisation tendancielle des régimes de sécurité sociale. La financiarisation est alors stimulée par l'endettement public.

Si la communauté nationale devient, comme l'écrit C. Marazzi, "une multitude de cas singuliers concrets", on peut alors rendre compte de la privatisation et de l'individualisation en terme de gouvernementalité de la façon suivante : la biopolitique étatique du fordisme est remplacée par une gouvernementalité financière marchande, l'individu se trouvant en situation de dépendance vis-à-vis du système financier. Via les crédits et les placements éventuels des organismes de retraite, des assurances et de l'épargne salariale, il entre dans une logique de financiarisation qui va se manifester par des contraintes qui vont peser sur sa vie (présente et future) et par certaines stratégies qu'il devra assumer. Ces contraintes l'amènent à se préoccuper de monnayer sa capacité créatrice et à assurer sa solvabilité, présente et future. Elles placent également le salarié plus particulièrement en situation de schizophrénie.

\section{c- La finance, nouvel exode du capital et nouvelle figure de l'antagonisme.}

Nous avons écrit auparavant que a déstabilisation financière n'est pas postérieure à la crise $\mathrm{du}$ fordisme et qu'elle fait partie de la crise du fordisme. L'analyse que propose A. Négri complète et éclaire ce qui a été dit.

Pour lui, ce qui est premier dans l'histoire de cette longue transition vers le postfordisme, c'est la nouvelle subjectivité et le comportement des travailleurs. Cela est dans l'ordre du positif et du dépassement. C'est le facteur de crise du fordisme (devenu moins maitrisable), et c'est aussi l'acte créateur qui enclenche un processus de reconfiguration. L'acte inaugural primitif est donc de ce côté là. L'acte financier est alors considéré comme une riposte. La décision de 1979 est une réponse politique du capital, alors en voie d'euthanasie, aux "désordres" du fordisme. Elle est par conséquent seconde dans la séquence des événement qui marquent l'inauguration de la période postfordiste, quoique étant aussi inaugurale à un certain niveau. A. Négri n'aborde pas la crise du fordisme principalement à partir des facteurs économiques. La réalisation et le dépassement de la crise sont abordés en termes politiques. Ceci est cohérent avec ce que nous avons écrit.

Ce qui est important c'est l'interprétation que nous tirons de cette interprétation quant au statut du capital financier dans le postfordisme : la finance peut être comprise comme l'exode du capital. Celui-ci ne s'implique plus dans l'industrie en ce sens qu'il ne joue pas les durées longues. Il joue le jeu des marchés financiers et acquiert une mobilité et une plasticité considérable, évitant ainsi l'immobilisation prolongée. Via la finance, le capital parvient à 
s'approprier de la plus value alors même que les règles habituelles de la production et de la concurrence se transforment radicalement. Le capital s'est mis à bonne distance du "réel" et une forme de scission est consommée. L'antagonisme entre le capital et le travail prend une nouvelle figure.

\section{$\underline{\text { d- La finance est l'évaluation du commun }}$}

La finance remplit plusieurs fonctions dont celle d'être une représentation intégrant des jugements sur le futur. Dans le postfordisme, la finance est en position d'évaluer une capacité de production qui est partagée et coopérative, c'est-à-dire commune, et projetée dans l'avenir. D'autre part, compte tenu de la liberté de mouvement des capitaux sur les marchés globaux, à une production qui se globalise, directement ou indirectement, souvent de façon complexe, correspond un système d'évaluation lui aussi globalisé.

L'incertitude importante a conduit à la création de moyens de gestion du risque dont les produits dérivés sont les plus connus. L'efficacité de cette socialisation du risque par le marché est à la mesure de la clarté et de la compréhension, par tous les acteurs, des informations communiquées sur le substrat des produits.

Les marchés financiers fonctionnent à partir d'évaluations qui sont de l'ordre du jugement, de l'opinion ou encore, selon A. Orlean, de la croyance. Ils n'ont pas pour vocation, en fait, de produire des représentations fidèles. La sphère financière produit essentiellement des jugements et des discours de justification qui ne reflètent d'ailleurs pas (ou plus) des valeurs ancrées dans le "réel". D'une part parce que les évaluations représentent pour partie les performances futures. Ensuite parce qu'il y a une crise de la mesure de la valeur. Celle-ci s'explique, notamment, par la créativité et par la difficulté d'identifier les facteurs de productivité, c'est-à-dire par la difficile traçabilité des sources de productivité. Seuls des jugements globaux peuvent s'exprimer et se réaliser.

Les discours de la finance ont ainsi une portée politique. D'abord ils mettent en jeu des autorités et des experts et les managers de la finance, c'est-à-dire des personnes "autorisées". Ensuite ils visent à créer de la confiance et à diffuser des opinions. Ils interviennent sur les comportements. Les marchés financiers sont des marchés d'opinion qui ne fonctionnent pas de façon démocratique puisque ce sont les intermédiaires et les acteurs professionnels qui monopolisent les leviers d'action et l'espace médiatique.

Si le langage de la finance est aussi, en tant que langage, un commun, force est de constater qu'il est devenu source d'exploitation. La prise en compte de valeurs extrafinancières dans les évaluations boursières ${ }^{20}$ est l'une des façons de reprendre en partie le contrôle de ce langage.

Paris, le 22 novembre 2007

\footnotetext{
${ }^{20}$ Considérations éthiques, de développement durable, de responsabilité sociale etc.
} 